\title{
Preoperative lymphocyte-to-monocyte ratio represents a superior predictor compared with neutrophil-to-lymphocyte and platelet- to-lymphocyte ratios for colorectal liver-only metastases survival
}

This article was published in the following Dove Press journal:

OncoTargets and Therapy

27 July 2017

Number of times this article has been viewed

\author{
Jianhong Pengl,* \\ Hui $\mathrm{Li}^{2}, *$ \\ Qingjian $\mathrm{Ou}^{1, *}$ \\ Junzhong Lin' \\ Xiaojun $\mathrm{Wu}^{\prime}$ \\ Zhenhai Lu' \\ Yunfei Yuan' \\ Desen Wan' \\ Yujing Fang' \\ Zhizhong Pan' \\ 'Department of Colorectal Surgery, \\ State Key Laboratory of Oncology in \\ South China, Collaborative Innovation \\ Center for Cancer Medicine, Sun \\ Yat-sen University Cancer Center, \\ Guangzhou, People's Republic of \\ China; ${ }^{2}$ Zhongshan School of Medicine, \\ Sun Yat-sen University, Guangzhou, \\ People's Republic of China \\ *These authors contributed equally \\ to this work
}

\begin{abstract}
Systemic inflammation was recognized as an essential factor contributing to the development of malignancies. This study aimed to investigate the prognostic value of preoperative lymphocyte-to-monocyte ratio (LMR), neutrophil-to-lymphocyte ratio (NLR), and platelet-to-lymphocyte ratio (PLR) in patients with colorectal liver-only metastases (CLOM) undergoing hepatectomy. We retrospectively enrolled 150 consecutive patients with CLOM between 2000 and 2012. The optimal cutoff values of continuous LMR, NLR, and PLR were determined using the receiver operating characteristic curve analysis. Recurrence-free survival (RFS) and overall survival (OS) related to the LMR, NLR, and PLR were analyzed using both Kaplan-Meier and multivariate Cox regression methods. Elevated LMR $(\geq 2.82)$ and lower NLR $(<4.63)$ were significantly associated with better RFS and OS in patients with CLOM after hepatectomy, instead of lower PLR $(<150.17)$. Multivariate Cox analysis identified elevated LMR as the only independent inflammatory factor for better RFS (hazard ratio, $0.591 ; 95 \%$ CI, 0.32-0.844; $P=0.008$ ) and OS (hazard ratio, $0.426 ; 95 \% \mathrm{CI}, 0.254-0.716 ; P=0.001$ ). In the subgroup analysis, elevated LMR was a significant favorable factor in both 5-year RFS and OS of patients with male gender, lymph node metastases, colon cancer, liver tumor with the largest diameter $<5 \mathrm{~cm}$, preoperative carcinoembryonic antigen level $<200 \mathrm{ng} / \mathrm{mL}$, negative hepatitis B virus infection, non-anatomic liver resection, postoperative chemotherapy, and non-preoperative chemotherapy. This study demonstrated that the preoperative LMR was an independent predictor of RFS and OS in patients with CLOM undergoing hepatic resection, and it appeared to be superior to the NLR and PLR.
\end{abstract}

Keywords: colorectal cancer, liver metastases, lymphocyte-to-monocyte ratio, survival

\section{Introduction}

With increasing incidence and mortality, colorectal cancer (CRC) has become the third leading cause of cancer deaths with an estimated mortality of 191,000 in People's Republic of China, in 2015. ${ }^{1}$ The development of distantly metastatic disease is the major cause of death, in which the liver is usually the most frequent site of metastatic disease. Approximately 20\%-25\% patients were initially diagnosed as having synchronous metastases and approximately half of the cases developed the metachronous disease after resecting the primary tumor. ${ }^{2,3}$ Hepatic resection is known to represent the only chance of curative treatment for colorectal liver-only

\footnotetext{
Correspondence: Zhizhong Pan; Yujing Fang

Department of Colorectal Surgery, Cancer Center, Sun Yat-sen University, 65I Dongfeng Road East, Guangzhou 510060, People's Republic of China Tel +86 2087343124 Fax +86 2087343637 Email panzhzh@sysucc.org.cn; fangyj@sysucc.org.cn
} 
metastases (CLOM). ${ }^{4,5}$ Unfortunately, a large proportion of the population with the disease fails to achieve favorable long-term survival due to tumor recurrence. ${ }^{6,7}$ Thus, identifying relevant prognostic factors can readily screen out the high-risk subgroups and subsequently optimize therapeutic strategies for CLOM.

To date, tumor pathologic staging, differentiation, and types have been widely used for predicting CRC outcomes of patients. ${ }^{8,9}$ In addition to tumor factors, systemic inflammation has been recognized as an essential factor contributing to the development of malignancies. ${ }^{10}$ Recently, growing evidence elucidated that a systemic inflammatory response was associated with poor clinical outcomes in numerous cancers. ${ }^{11,12}$ Specifically, among multiple systemic inflammatory markers, the lymphocyte-to-monocyte ratio (LMR), neutrophil-to-lymphocyte ratio (NLR), and platelet-tolymphocyte ratio (PLR) have been successfully identified as prognostic predictors for patients with advanced CRC receiving chemotherapy or surgical procedure. ${ }^{13-15}$ However, evidence for the prognostic predictive values of preoperative LMR, NLR, and PLR in patients with CLOM receiving hepatic resection remains limited. ${ }^{16,17}$ Therefore, this study hypothesized that some of these inflammatory indexes might exhibit a potential and effective prognostic value for CLOM after hepatic resection.

This study aimed to investigate the prognostic impact of preoperative LMR, NLR, and PLR in patients with CLOM undergoing hepatic resection. Moreover, it also aimed to find out an authentic biomarker for accurate prognostic prediction by comparing the prognostic values among these systemic inflammatory biomarkers.

\section{Methods}

\section{Patients}

This study retrospectively enrolled 150 patients from September 2000 to July 2012 at Sun Yat-sen University Cancer Center, People's Republic of China, undergoing primary tumor resection and hepatectomy with curative intent. The enrolled patients met the following inclusion criteria: 1) histologically confirmed colorectal adenocarcinoma, 2) metastases limited to liver, 3) R0 or R1 resection, and 4) no percutaneous ablation. Patients were excluded from the analysis if they had other active malignancies, died in the postoperative period, or had missing preoperative data making it impossible to calculate the LMR, NLR, and PLR. Patient demographics, primary and metastatic tumor characteristics, preoperative treatment, and follow-up results were reviewed in detail from the medical records and the follow-up system of Sun Yat-sen University Cancer Center. All procedures performed in studies involving human participants were in accordance with the 1964 Helsinki Declaration and its later amendments or comparable ethical standards. Institutional Review Board approval was also obtained from the independent ethics committee at the Sun Yat-sen University Cancer Center. Informed consent was waived in this noninterventional, observational, and retrospective study, in which the patient data were kept strictly confidential.

\section{Treatments}

The treatment strategy for every patient was determined according to the final agreement of the multidisciplinary team, including staff from the Department of Colorectal Surgery, Hepatobiliary Surgery, Medical Oncology, and Medical Imaging and Invasive Technology. The operability of liver metastases was assessed using an enhanced chest computed tomography and abdominal and pelvic nuclear magnetic resonance imaging. Patients whose tumors were deemed to be both resectable and low-risk recurrent were suggested to undergo surgery directly, while those considered as potentially resectable or high-risk recurrent were suggested to perform preoperative chemotherapy followed by tumor resection.

\section{Inflammatory index calculation}

The systemic inflammatory data (neutrophil, lymphocyte, monocyte, and platelet counts) were collected from the blood routine test between 8 and 10 am within 7 days before the hepatic operation. LMR was calculated by dividing the absolute number of circulating lymphocytes by the absolute number of monocytes. Likewise, the NLR or PLR was generated by dividing the absolute counts of circulating neutrophils or platelets by the absolute number of lymphocytes.

\section{Follow-up}

All patients were suggested to return for a subsequent visit every 3 months for 2 years and then semi-annually until 3 years after hepatic resection. The evaluation included clinical examination, carcinoembryonic antigen (CEA) level, abdominal ultrasonography, and chest radiograph. Chest computed tomography, abdominal/pelvic magnetic resonance imaging, and colonoscopy were performed annually. Overall survival (OS) was defined as the time length from operation to death for any cause, while recurrence-free survival (RFS) was defined as the interval from hepatic resection to disease recurrence or death. The last follow-up visit occurred in September 2015. 


\section{Statistical analysis}

The receiver operating characteristic (ROC) curve analysis was applied to calculate the area under the ROC curve (AUC) and then determine the optimal cutoff values of continuous LMR, NLR, and PLR according to the 5-year OS. The correlation between clinical-pathologic parameters and LMR, NLR, and PLR was assessed by the chi-square test or Fisher's exact test, as appropriate. Survival outcomes for the binary level of inflammatory indexes were compared using the Kaplan-Meier log-rank test. Potential effects of clinical variables on RFS and OS were examined using univariate Cox proportional hazards. Variables proving statistical significance in univariate Cox models were further assessed using multivariate Cox models. The multivariate Cox proportional hazard model was used to further identify independent prognostic factors for RFS and OS. The hazard ratios (HRs) and CIs were subsequently calculated. The statistical analysis and forest plot figure were performed using the IBM SPSS Statistics 22 software (IBM Corporation, Armonk, NY, USA) and GraphPad Prism version 6.01 (GraphPad Software, Inc, La Jolla, CA, USA), respectively. All statistical tests used in this study were two sided, and a $P$-value of $<0.05$ was considered as statistically significant.

\section{Results}

\section{Optimal cutoff determination}

The AUC of LMR, NLR, and PLR was 0.639 (95\% CI, $0.550-0.728, P=0.004$ ), 0.568 (95\% CI, 0.477-0.660, $P=0.152$ ), and 0.518 (95\% CI, 0.424-0.611, $P=0.707$ ), respectively (Figure 1). According to the highest Youden index (specificity + sensitivity-1), the optimal cutoff values chosen for the LMR, NLR, and PLR were 2.82, 4.63, and 150.17, respectively ( 0.27 for LMR, 0.13 for NLR, and 0.10 for PLR). In the subsequent analysis, the LMR, NLR, and PLR were dichotomized into high-level and low-level groups according to the optimal cutoff values.

\section{Patient characteristics}

Of the total patients, $64.7 \%(97 / 150)$ were male and $35.3 \%$ $(53 / 150)$ were female, with a median age of 58 years (range 20-82). Among the patients with the primary tumor, $58 \%$ had colon cancer, while $42 \%$ had rectum cancer. In 84 (59.2\%) patients, the primary tumors involved lymph node metastasis. The majority of patients $(85 / 150,56.7 \%)$ presented a solitary tumor, and $62.7 \%(94 / 150)$ had synchronous metastases at the time of diagnosis. As for the hepatic metastases, the median size of the largest tumor was $2.8 \mathrm{~cm}$ (range $0.3-12.1 \mathrm{~cm})$. In addition, 46.7\% (70/150) patients

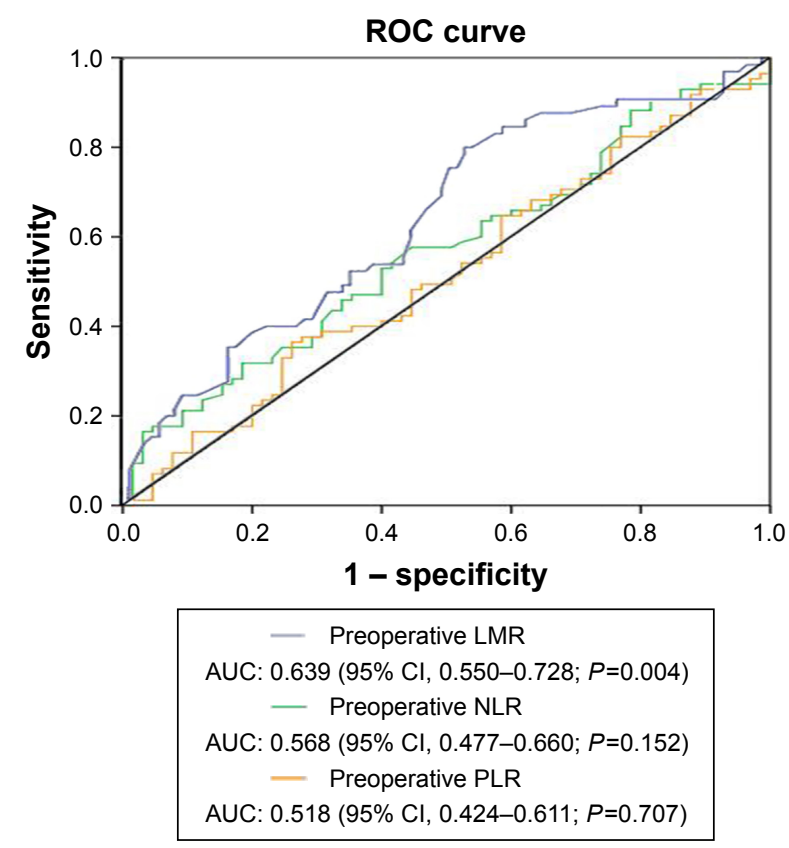

Figure I ROC curve for the preoperative LMR, NLR, and PLR according to the 5-year OS.

Abbreviations: AUC, area under the curve; LMR, lymphocyte-to-monocyte ratio; NLR, neutrophil-to-lymphocyte ratio; OS, overall survival; PLR, platelet-tolymphocyte ratio; ROC, receiver operating characteristic.

received synchronous resection for primary tumor and liver metastases. With respect to chemotherapy, 59 (39.3\%) patients received neoadjuvant chemotherapy before hepatectomy and $110(73.3 \%)$ patients received postoperative chemotherapy. The detailed characteristics of the patients in this study are described in Table 1.

\section{Relationships between preoperative LMR, NLR, and PLR and patient characteristics}

A larger proportion of patients with an elevated LMR showed a lower preoperative CEA level compared with that of the LMR-low group ( $92.8 \%$ vs $79.2 \%, P=0.048$ ), as shown in Table 2. Additionally, a higher proportion of the LMRhigh group received postoperative chemotherapy $(82.5 \%$ vs $56.6 \%, P<0.001)$. Patients in the NLR-high group were more likely to present multiple hepatic metastases than those in the NLR-low group ( $68.8 \%$ vs $40.3 \%, P=0.030$ ). In the PLR-high group, $77.1 \%$ patients had a negative hepatic resection margin, while in the PLR-low group, only $52.9 \%$ patients had a negative resection margin $(P=0.005)$. As to the different LMR, NLR, and PLR subgroups, clinical characteristics including gender distribution, lymph node metastases, primary tumor location, largest hepatic tumor size, metastatic tumor distribution, timing of metastasis, preoperative chemotherapy, and liver resection were comparable. 
Table I Basic characteristics of total patients with colorectal cancer liver-only metastases

\begin{tabular}{|c|c|}
\hline Characteristics & $\begin{array}{l}\text { Total patients } \\
(n, \%)\end{array}$ \\
\hline Age (years), range & $58(20-82)$ \\
\hline \multicolumn{2}{|l|}{ Gender $(n=150)$} \\
\hline Male & $97(64.7)$ \\
\hline Female & $53(35.3)$ \\
\hline \multicolumn{2}{|l|}{ Preoperative chemotherapy $(n=150)$} \\
\hline No & $91(60.7)$ \\
\hline Yes & $59(39.3)$ \\
\hline \multicolumn{2}{|l|}{ Preoperative HBV infection $(n=146)$} \\
\hline Negative & $128(87.7)$ \\
\hline Positive & $18(12.3)$ \\
\hline \multicolumn{2}{|l|}{ Preoperative CEA level $(n=\mid 44), n g / m L$} \\
\hline$<200$ & $132(91.7)$ \\
\hline$\geq 200$ & $12(8.3)$ \\
\hline Preoperative LMR (median, range) & $3.33(0.16-11.0)$ \\
\hline Preoperative NLR (median, range) & $2.0(0.6-82.0)$ \\
\hline Preoperative PLR (median, range) & $|23.7|(39.05-990.0)$ \\
\hline \multicolumn{2}{|l|}{ Timing of metastasis $(n=150)$} \\
\hline Synchronous & $94(62.7)$ \\
\hline Metachronous & $56(37.3)$ \\
\hline Number of metastatic tumors (median, range) & $I(I-I I)$ \\
\hline Largest tumor size $(\mathrm{cm})$, range & $2.8(0.3-12.1)$ \\
\hline \multicolumn{2}{|l|}{ Tumor distribution $(n=150)$} \\
\hline Unilobar & $104(69.3)$ \\
\hline Bilobar & $46(30.7)$ \\
\hline \multicolumn{2}{|l|}{ Liver resection $(n=\mid 50)$} \\
\hline Anatomic & $19(12.7)$ \\
\hline Non-anatomic & $|3|(87.3)$ \\
\hline \multicolumn{2}{|l|}{ Resection margin $(n=150)$} \\
\hline Negative & 91 (60.7) \\
\hline Positive & $59(39.3)$ \\
\hline \multicolumn{2}{|l|}{ Postoperative chemotherapy $(n=\mid 50)$} \\
\hline No & $40(26.7)$ \\
\hline Yes & $110(73.3)$ \\
\hline
\end{tabular}

Abbreviations: CEA, carcinoembryonic antigen; HBV, hepatitis B virus; LMR, lymphocyte-to-monocyte ratio; NLR, neutrophil-to-lymphocyte ratio; PLR, plateletto-lymphocyte ratio.

\section{Survival outcomes and inflammatory index}

The median follow-up period for all the patients was 36 months (range 2-126 months). During the follow-up period, 68.0\% $(102 / 150)$ patients experienced tumor recurrence, including $65.7 \%(67 / 102)$ patients with intrahepatic recurrence, $13.7 \%$ (14/102) patients with lung metastases, $8.8 \%(9 / 102)$ patients with abdominal pelvic metastases, and $11.8 \%(12 / 102)$ patients with multiple organ metastases. As a result, $56.7 \%$ (85/150) patients died of disease progression. Regarding the entire study population, the 3- and 5-year RFS rates were $39.4 \%$ and $35.4 \%$, respectively, and the 3 - and 5-year OS rates were $56.8 \%$ and $43.2 \%$, respectively. Patients with an elevated LMR had significantly better 5-year RFS and OS compared with those of the LMR-low group (RFS: $43.1 \%$ vs $21.4 \%$,
$P=0.001$; OS: $52.2 \%$ vs $27.5 \%, P<0.001$; Figure $2 \mathrm{~A}$ and D). On the contrary, the estimated 5-year RFS and OS for the NLR-high group were significantly inferior to those of NLR-low group (RFS: $12.5 \%$ vs $38.5 \%, P=0.015$; OS: $18.8 \%$ vs $46.7 \%, P=0.004$; Figure $2 \mathrm{~B}$ and $\mathrm{E}$ ). Regarding the PLR groups, neither the 5-year RFS rates (36.3\% vs $33.3 \%$, $P=0.593$; Figure $2 \mathrm{C}$ ) nor the 5 -year OS rates $(44.4 \%$ vs $40.4 \%$, $P=0.229$; Figure $2 \mathrm{~F}$ ) showed any statistical difference.

The univariate analysis revealed that the high LMR was associated with better RFS (HR, 0.523; 95\% CI, 0.35-0.782; $P=0.002)$ and $\mathrm{OS}(\mathrm{HR}, 0.475 ; 95 \% \mathrm{CI}, 0.31-0.728 ; P=0.001$ ), as shown in Table 3. On the contrary, high NLR, lymph node metastases, number of metastatic tumors $>1$, largest metastatic tumor size $\geq 5 \mathrm{~cm}$, and bilobar tumor distribution were correlated with both worse RFS and OS. Additionally, patients receiving preoperative chemotherapy showed poorer RFS than those without preoperative treatment (HR, 1.665; 95\% CI, 1.113-2.491; $P=0.013$ ).

The multivariate analysis subsequently showed that lymph node metastases (HR, 1.785; 95\% CI, 1.139-2.797; $P=0.011)$, largest metastatic tumor size $\geq 5 \mathrm{~cm}$ (HR, 1.928; 95\% CI, 1.217-3.054; $P=0.005$ ), and high LMR (HR, $0.591 ; 95 \%$ CI, $0.32-0.844 ; P=0.008$ ) were meaningful prognostic factors for RFS. Similarly, lymph node metastases (HR, 2.271; 95\% CI, 1.382-3.731; $P=0.001$ ), largest metastatic tumor size $\geq 5 \mathrm{~cm}$ (HR, 1.754; 95\% CI, 1.061-2.901; $P=0.028$ ), and high LMR (HR, 0.426 ; 95\% CI, 0.254-0.716; $P=0.001$ ) were also defined as independent factors for OS.

The LMR in the subgroups stratified by 14 clinical parameters was subjected to the univariate Cox model analysis to further verify the prognostic value of LMR. The prognostic value of LMR was consistent in both 5-year RFS and OS when considering the subgroups by age, tumor number, or distribution. In addition, the prognostic value of LMR was consistent in 5-year OS when considering the subgroups by timing of metastasis and resection margin (Figure 3). Thus, high LMR was demonstrated as the favorable prognostic factor for patients with male gender, lymph node metastases, colon cancer, metastatic tumor with the largest diameter $<5 \mathrm{~cm}$, preoperative CEA level $<200 \mathrm{ng} / \mathrm{mL}$, negative hepatitis $\mathrm{B}$ virus infection, non-anatomic liver resection, postoperative chemotherapy, and non-preoperative chemotherapy in both 5-year RFS and OS.

\section{Discussion}

To date, mounting studies have mainly concentrated on the biologic behavior and presentation of tumor histopathologic parameters to screen out feasible cancer-related variables 
Table 2 Clinical characteristics of the patients stratified by LMR, NLR, and PLR

\begin{tabular}{|c|c|c|c|c|c|c|c|c|c|}
\hline \multirow[t]{3}{*}{ Characteristics } & \multicolumn{3}{|l|}{ LMR } & \multicolumn{3}{|l|}{ NLR } & \multicolumn{3}{|l|}{ PLR } \\
\hline & $<\mathbf{2 . 8 2}$ & $\geq 2.82$ & $P$-value & $<4.63$ & $\geq 4.63$ & $P$-value & $<\mathbf{1 5 0 . 1 7}$ & $\geq 150.17$ & $P$-value \\
\hline & $n=53, n(\%)$ & $n=97, n(\%)$ & & $n=134, n(\%)$ & $n=16, n(\%)$ & & $n=102, n(\%)$ & $n=48, n(\%)$ & \\
\hline Gender & & & 0.183 & & & 0.718 & & & 0.455 \\
\hline Male & 38 (7I.7) & $59(60.8)$ & & $86(64.2)$ & II (68.8) & & $68(66.7)$ & $29(60.4)$ & \\
\hline Female & $15(28.3)$ & $38(39.2)$ & & $48(35.8)$ & $5(3 \mid .2)$ & & $34(33.3)$ & $19(39.6)$ & \\
\hline Age, years & & & 0.896 & & & 0.734 & & & 0.500 \\
\hline$<58$ & $24(45.3)$ & $45(46.4)$ & & $6 \mathrm{I}(45.5)$ & $8(50.0)$ & & $45(44.1)$ & $24(50.0)$ & \\
\hline$\geq 58$ & $29(54.7)$ & $52(53.6)$ & & $73(54.5)$ & $8(50.0)$ & & $57(55.9)$ & $24(50.0)$ & \\
\hline Preoperative chemotherapy & & & 0.451 & & & 0.702 & & & 0.080 \\
\hline No & $30(56.6)$ & $61(62.9)$ & & $82(61.2)$ & $9(56.3)$ & & $57(55.9)$ & $34(70.8)$ & \\
\hline Yes & $23(43.4)$ & $36(37.1)$ & & $52(38.8)$ & $7(43.7)$ & & $45(44.1)$ & $14(29.2)$ & \\
\hline Preoperative HBV infection & & & 0.297 & & & $0.55 \mathrm{I}$ & & & 0.202 \\
\hline Negative & $42(79.2)$ & $86(88.7)$ & & II 5 (85.8) & $13(81.3)$ & & $88(86.3)$ & $40(83.3)$ & \\
\hline Positive & $9(17.0)$ & $9(9.3)$ & & $15(11.2)$ & $3(18.7)$ & & $10(9.8)$ & $8(16.7)$ & \\
\hline Unknown & $2(3.8)$ & $2(2.1)$ & & $4(3.0)$ & 0 & & $4(3.9)$ & 0 & \\
\hline Primary tumor location & & & 0.928 & & & 0.493 & & & 0.955 \\
\hline Colon & $31(58.5)$ & $56(57.7)$ & & $79(59.0)$ & $8(50.0)$ & & $59(57.8)$ & $28(58.3)$ & \\
\hline Rectum & $22(41.5)$ & $4 \mathrm{I}(42.3)$ & & $55(41.0)$ & $8(50.0)$ & & $43(42.2)$ & $20(4 \mid .7)$ & \\
\hline Lymph node metastasis & & & 0.155 & & & 0.584 & & & 0.348 \\
\hline No & $25(47.2)$ & $33(34.0)$ & & $5 I(38.1)$ & $7(43.7)$ & & $43(42.2)$ & $15(31.3)$ & \\
\hline Yes & $27(50.9)$ & $57(58.8)$ & & $75(56.0)$ & $9(56.3)$ & & $53(52.0)$ & $31(64.6)$ & \\
\hline Unknown & I (2.0) & $7(7.2)$ & & $8(6.0)$ & 0 & & $6(5.9)$ & $2(4.2)$ & \\
\hline Timing of metastasis & & & 0.066 & & & 0.595 & & & 0.075 \\
\hline Synchronous & $28(52.8)$ & $66(68.0)$ & & $83(61.9)$ & II (68.8) & & $59(57.8)$ & 35 (72.9) & \\
\hline Metachronous & $25(47.2)$ & $31(32.0)$ & & $51(38.1)$ & $5(3 \mid .2)$ & & $43(42.2)$ & $13(27.1)$ & \\
\hline Number of metastatic tumors & & & 0.483 & & & 0.030 & & & 0.180 \\
\hline 1 & $28(52.8)$ & $57(58.8)$ & & $80(59.7)$ & $5(31.2)$ & & $54(52.9)$ & $31(64.6)$ & \\
\hline$>1$ & $25(47.2)$ & $40(4 \mid .2)$ & & $54(40.3)$ & $11(68.8)$ & & $48(47.1)$ & $17(35.4)$ & \\
\hline Largest tumor size, $\mathrm{cm}$ & & & 0.120 & & & 0.518 & & & 0.455 \\
\hline$<5$ & $36(67.9)$ & $77(79.4)$ & & $102(76.1)$ & II (68.8) & & $75(73.5)$ & $38(79.2)$ & \\
\hline$\geq 5$ & $17(32.1)$ & $20(20.6)$ & & $32(23.9)$ & $5(3 \mid .2)$ & & $27(26.5)$ & $10(20.8)$ & \\
\hline Tumor distribution & & & 0.309 & & & 0.076 & & & 0.302 \\
\hline Unilobar & $34(64.2)$ & $70(72.2)$ & & $96(71.6)$ & $8(50.0)$ & & $68(66.7)$ & $36(75.0)$ & \\
\hline Bilobar & $19(35.8)$ & $27(27.8)$ & & $38(28.4)$ & $8(50.0)$ & & $34(33.3)$ & $12(25.0)$ & \\
\hline Preoperative CEA level, $\mathrm{ng} / \mathrm{mL}$ & & & 0.048 & & & 0.680 & & & 0.558 \\
\hline$<200$ & $42(79.2)$ & $90(92.8)$ & & $119(88.8)$ & $13(81.3)$ & & $90(88.2)$ & $42(87.5)$ & \\
\hline$\geq 200$ & $7(13.2)$ & $5(5.2)$ & & $10(7.5)$ & $2(12.5)$ & & $9(8.8)$ & $3(6.3)$ & \\
\hline Unknown & $4(7.5)$ & $2(2.1)$ & & $5(3.7)$ & I (6.3) & & $3(2.9)$ & $3(6.3)$ & \\
\hline Liver resection & & & 0.509 & & & 0.107 & & & 0.966 \\
\hline Anatomic & $8(15.1)$ & II (II.3) & & $19(14.2)$ & 0 & & $13(12.7)$ & $6(12.5)$ & \\
\hline Non-anatomic & $45(84.9)$ & $86(88.7)$ & & $115(85.8)$ & $16(100.0)$ & & $89(87.3)$ & $42(87.5)$ & \\
\hline Resection margin & & & 0.146 & & & 0.214 & & & 0.005 \\
\hline Negative & $28(52.8)$ & $63(64.9)$ & & $79(59.0)$ & $12(75.0)$ & & $54(52.9)$ & $37(77.1)$ & \\
\hline Positive & $25(47.2)$ & $34(35.1)$ & & $55(4 I .0)$ & $4(25.0)$ & & $48(47.1)$ & II (22.9) & \\
\hline Postoperative chemotherapy & & & 0.001 & & & 0.102 & & & 0.635 \\
\hline No & $23(43.4)$ & $17(17.5)$ & & $33(24.6)$ & $7(43.8)$ & & $26(25.5)$ & $14(29.2)$ & \\
\hline Yes & $30(56.6)$ & $80(82.5)$ & & $101(75.4)$ & $9(56.2)$ & & $76(74.5)$ & $34(70.8)$ & \\
\hline
\end{tabular}

Abbreviations: CEA, carcinoembryonic antigen; HBV, hepatitis B virus; LMR, lymphocyte-to-monocyte ratio; NLR, neutrophil-to-lymphocyte ratio; PLR, platelet-tolymphocyte ratio.

for predicting the prognosis of patients with CLOM after hepatic resection. ${ }^{18-20}$ However, these variables did not entirely present the complete potency for predicting and guiding the individual treatment appropriately. Recently, growing evidence suggested that systemic inflammation potentially contributed to the tumor development, and it is now considered as a hallmark of CRC. ${ }^{21-23}$ Therefore, this study evaluated the prognostic value of three common inflammation-based indexes (LMR, NLR, and PLR) and determined whether they could serve as alternative items 

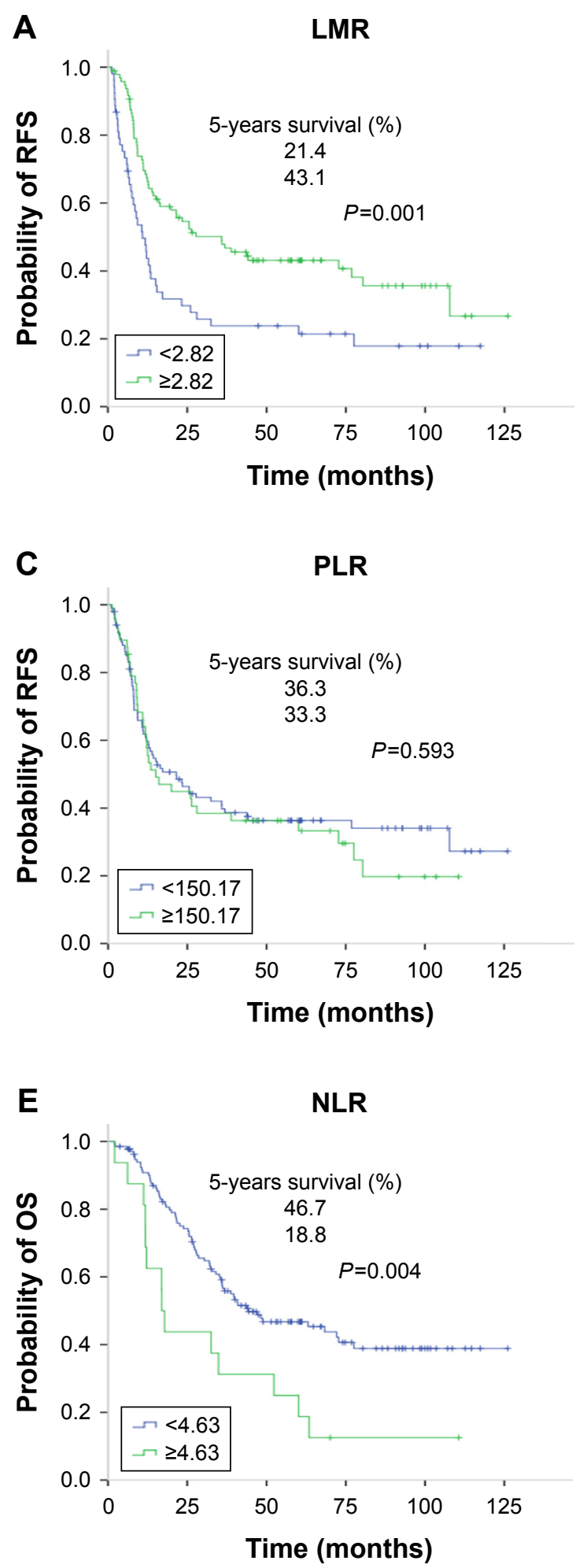

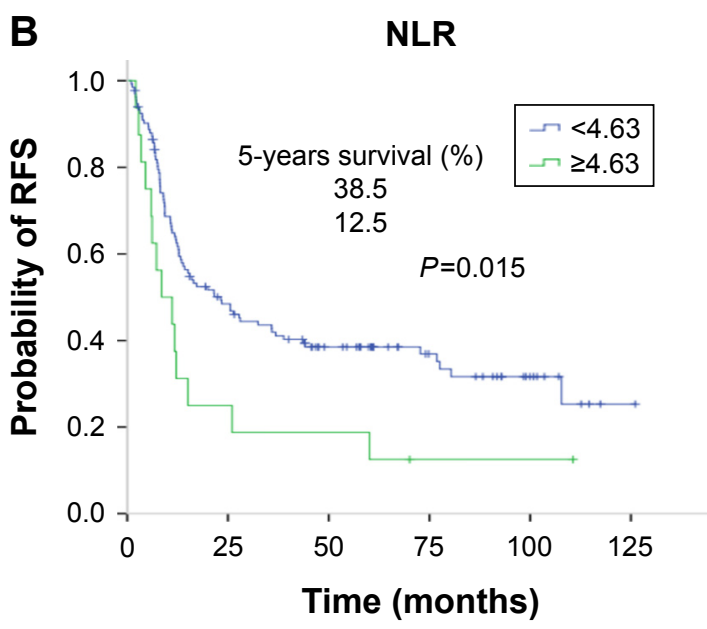

D LMR
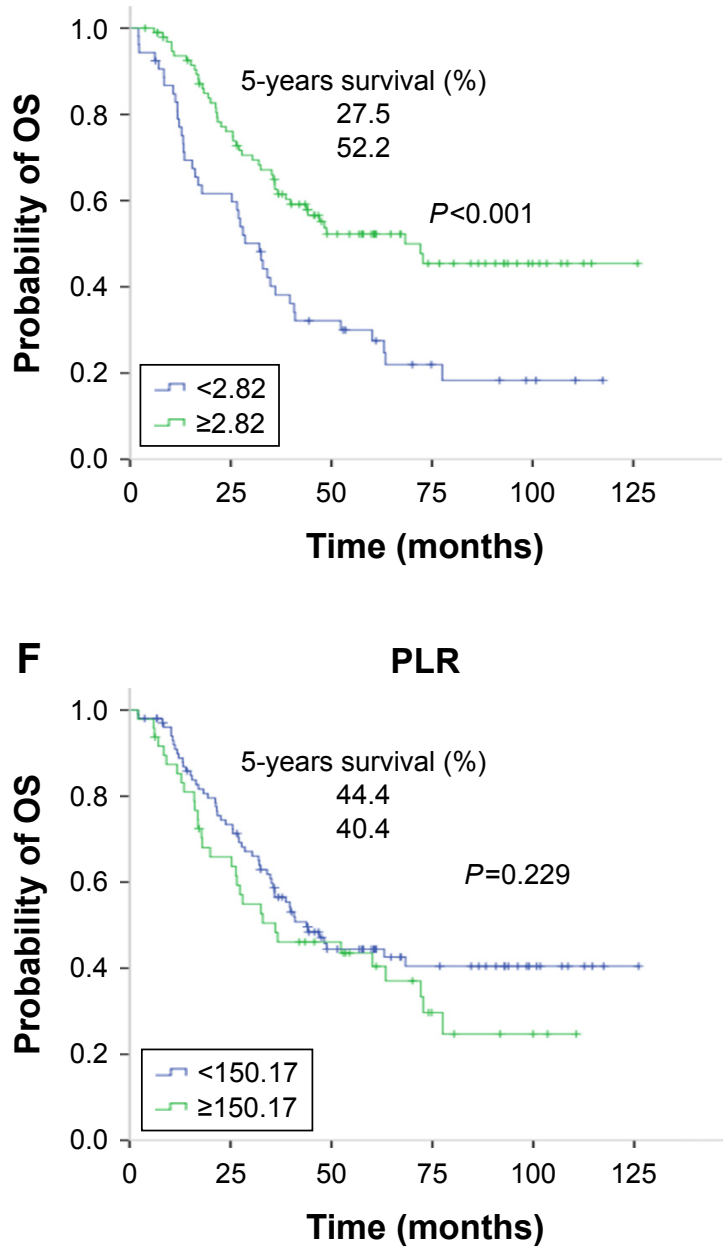

Figure 2 Kaplan-Meier curves of LMR, NLR, and PLR for comparing the 5-year RFS and OS.

Notes: A, B, and C are the survival curves of LMR, NLR and PLR for RFS, respectively; D, E, and F are the survival curves of LMR, NLR, and PLR for OS, respectively. Abbreviations: LMR, lymphocyte-to-monocyte ratio; NLR, neutrophil-to-lymphocyte ratio; OS, overall survival; PLR, platelet-to-lymphocyte ratio; RFS, recurrence-free survival.

to tailor different risk subgroups for patients with CLOM receiving hepatic resection.

After prognostic comparison, the LMR exhibited the highest prognostic diagnostic value $(\mathrm{AUC}=0.639 ; 95 \% \mathrm{CI}$,
0.550-0.728; $P=0.004$ ) and was confirmed as a superior predictor of RFS and OS in patients with CLOM undergoing curative hepatectomy, compared with the NLR and PLR. Furthermore, the findings of this study also demonstrated that 


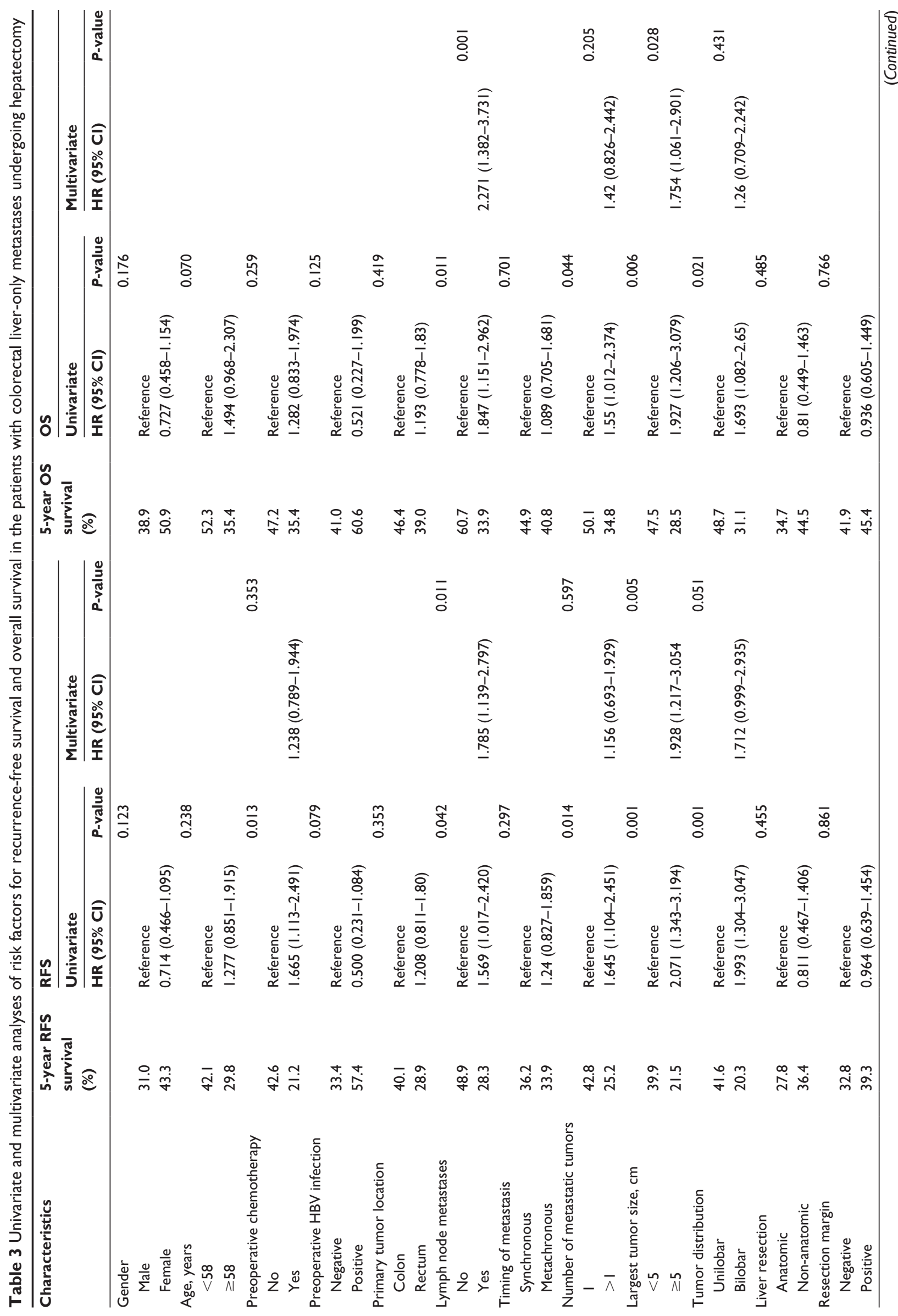




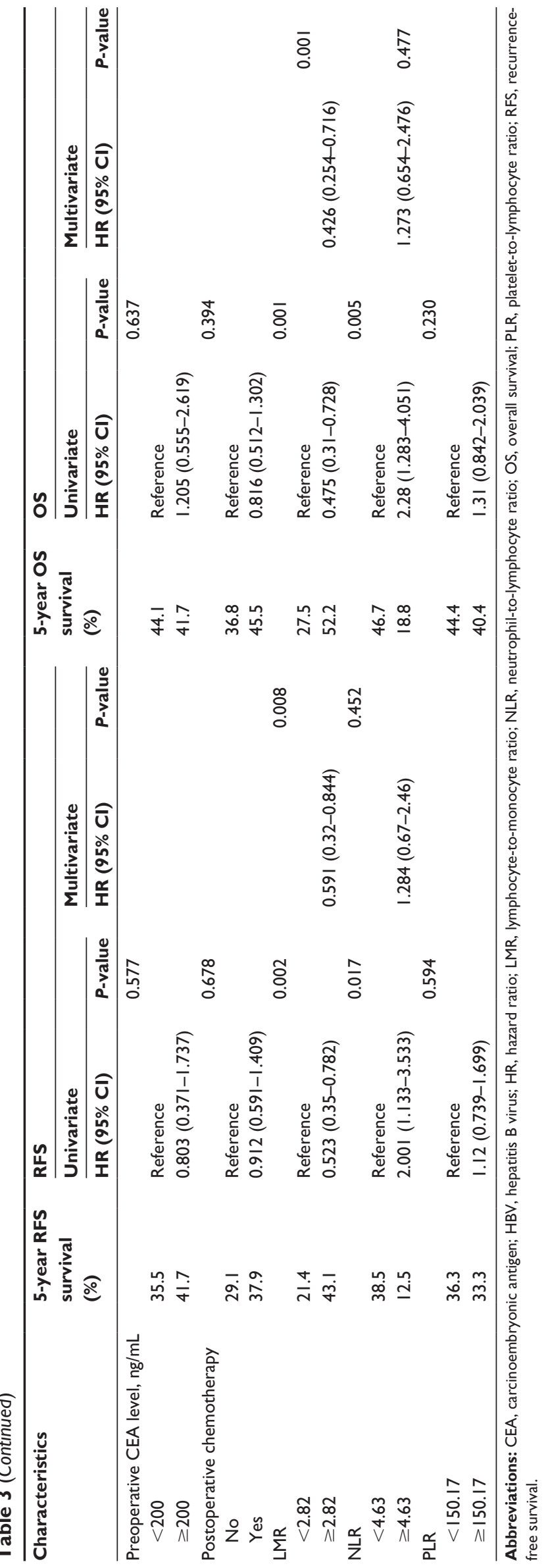

preoperative LMR was the only independent inflammatory predictor for both RFS and OS. Additionally, the high LMR predicted a favorable prognosis in patients with male gender, lymph node metastases, colon cancer, liver tumor with the largest diameter $<5 \mathrm{~cm}$, preoperative CEA level $<200 \mathrm{ng} / \mathrm{mL}$, negative hepatitis B virus infection, nonanatomic liver resection, postoperative chemotherapy, and non-preoperative chemotherapy. Overall, these proved the hypothesis of this study and suggested that systemic inflammatory biomarkers, such as LMR, might be the same alternative indicator to further stratify patients with CLOM, compared with the conventional histopathologic parameters.

So far, the predictive value of the preoperative LMR in CRC has been identified, and the decreased LMR delivered an unfavorable impact on the long-term survival among various stage subgroups..$^{24}$ Recently, one of the largest retrospective cohort studies revealed that the LMR appeared to be superior to the NLR, PLR, Glasgow prognostic score, BRAF mutation status, and mismatch repair status in prognostic prediction, which further indicated that the LMR was an independent predictor of OS in patients with nonmetastatic CRC undergoing curative resection. ${ }^{25}$ In patients with unresectable metastatic CRC who received palliative chemotherapy, previous studies have elucidated that the LMR was also a useful marker for predicting the long-term survival and the efficacy of chemotherapy ${ }^{26,27}$ As for the patients with CLOM undergoing curative liver resection, Neofytou et al first identified that the preoperative LMR remained the only independent prognostic factor for cancer-specific survival, compared with the NLR and PLR. ${ }^{17}$ However, unlike the results of this study, the LMR was not proven an independent prognostic factor for RFS in their study. As a matter of fact, the recruited patients totally received preoperative chemotherapy in the study of Neofytou et al, while only $39.3 \%$ patients received the adjuvant therapy in this study. Consistent with the results of Neofytou et al's study, high LMR was not significantly associated with better 5-year RFS of the patients receiving preoperative chemotherapy in this study (HR, $0.583 ; 95 \% \mathrm{CI}$, 0.314-1.084; $P=0.088$; Figure 3). However, low LMR indicated worse survival outcome in non-preoperative chemotherapy subgroup. We considered that neoadjuvant chemotherapy contributed to improve the RFS of the LMR-low group, thus reducing the differences among survival outcomes. In addition, recurrent tumors in the lower-LMR group might be more resistant to additional chemotherapy than those in the higher-LMR group, leading to decreased OS, thus resulting in a significant OS difference in the two groups. ${ }^{28}$ Together, it is worth further exploring the prognostic value of LMR in the extended cohort of patients with CLOM. 


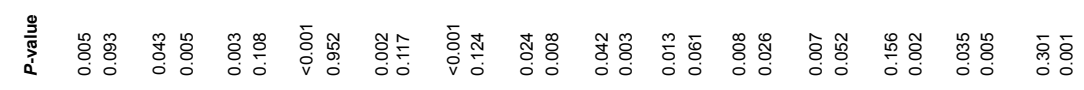

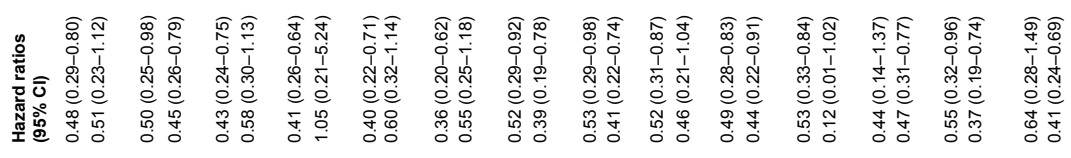

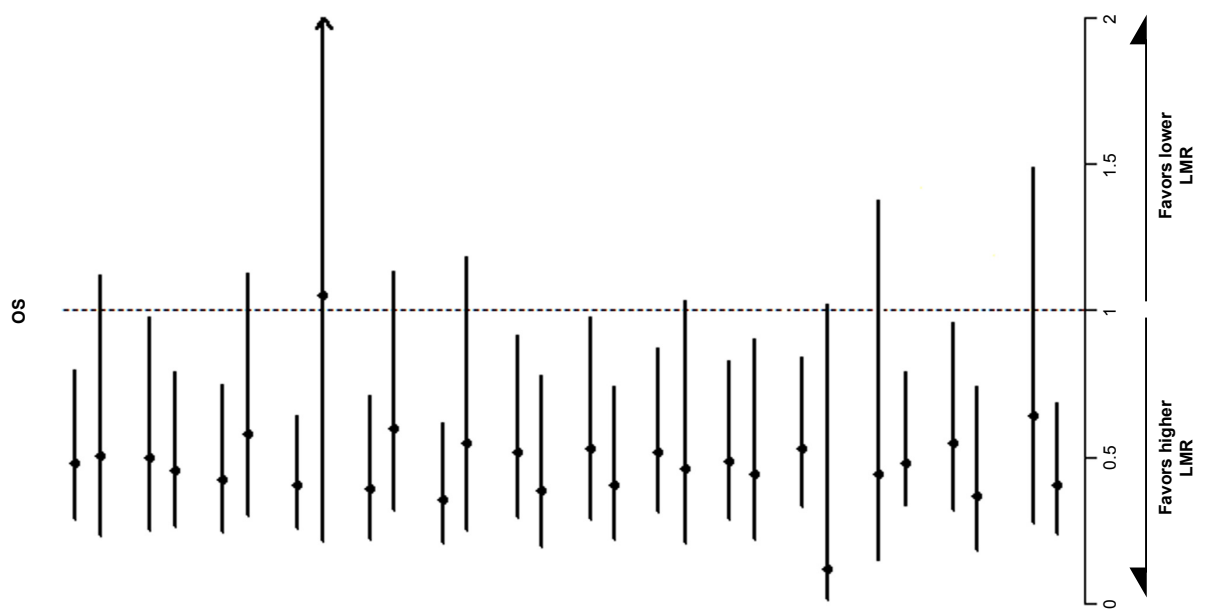

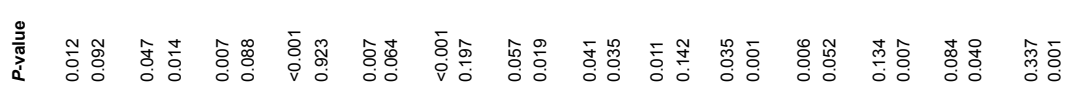

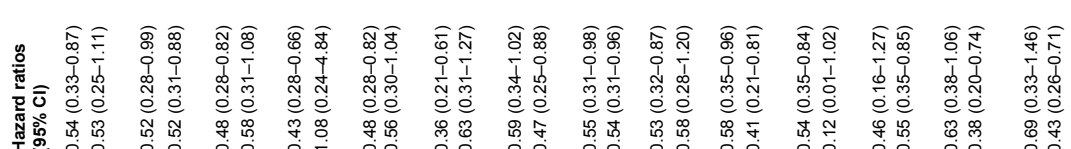

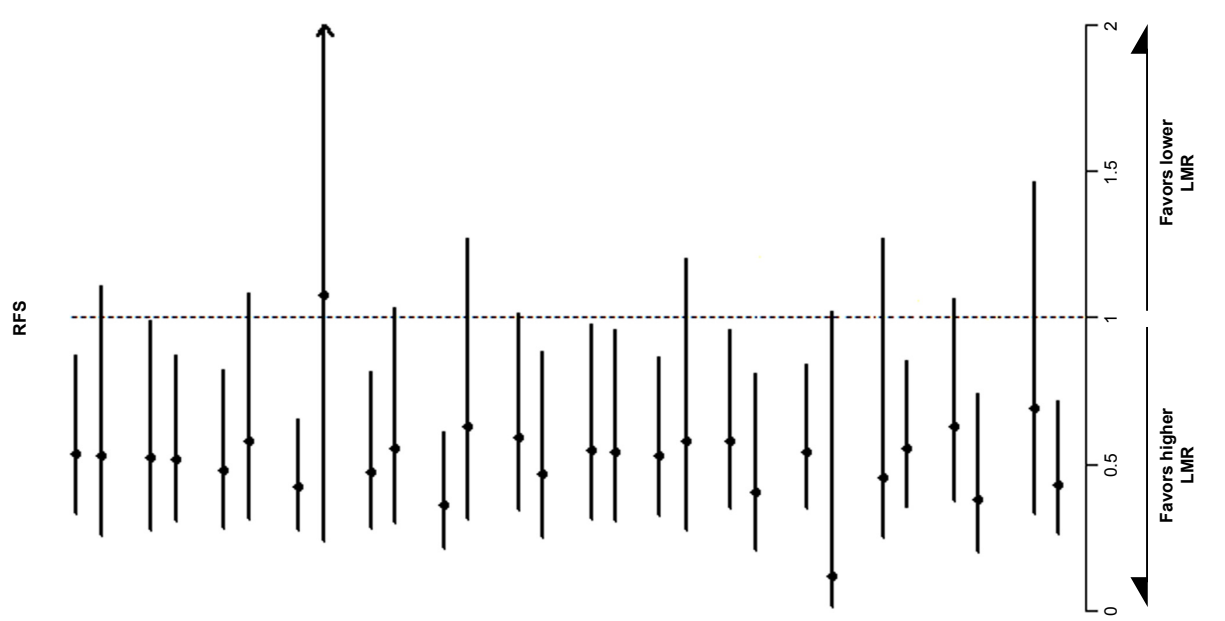

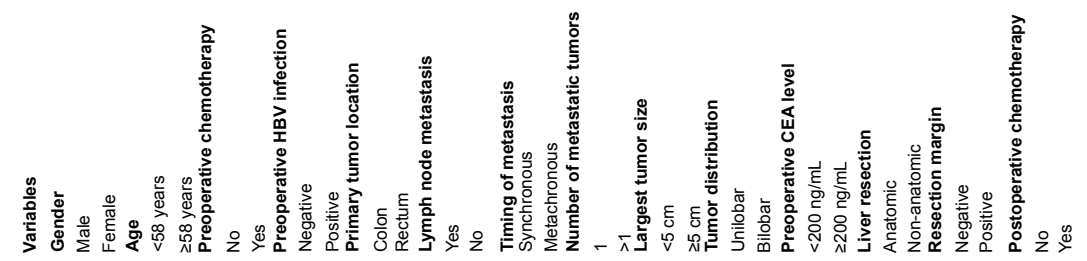


Several mechanisms could contribute to the outcome that the lower LMR compromised the long-term survival in patients with CLOM. The combination of two changes raised a very important role in the process: the relatively lower number of blood-circulating lymphocytes and excess monocytes. Previous studies have elucidated that lymphocytes played critical roles in the antitumor immunity of the host by infiltrating into the tumor microenvironment after being triggered by immunologic antitumor reaction. ${ }^{29,30}$ However, systemic inflammation significantly decreased cellular immunity, resulting in lymphocytopenia, which was marked as a decrease in $\mathrm{CD}^{+}$helper lymphocytes and an increase in $\mathrm{CD} 8^{+}$suppressor lymphocytes. ${ }^{31} \mathrm{~A}$ decreased number of circulating lymphocytes was, therefore, considered to be responsible for the insufficient immunologic reaction against the tumor, contributing to the poor cancer prognosis after surgical procedure in CRC. ${ }^{32}$ On the contrary, the excess circulating monocytes are recruited to the tumor sites and differentiated into macrophages, which become the tumor-associated macrophages (TAMs) ${ }^{33,34}$ It is generally accepted that the TAMs act like the "jack-of-all-trades", being involved mostly in protumoral functions, including tumor cell growth, angiogenesis, migration, invasion, metastasis, and immune system suppression. ${ }^{35,36}$ Growing clinical evidence showed that an increased number of TAMs was correlated with therapy failure and poor prognosis in cancer patients. ${ }^{37,38}$ As a result, elevated circulating serum levels of monocytes may reflect increased production of tissue TAMs, high tumor burden, and immunodepression, entirely leading to worse survival outcomes.

This is a novel study that demonstrated the LMR to be superior to the NLR and PLR as an independent predictor for both RFS and OS in patients with CLOM. Determining the preoperative LMR might provide useful information for the therapeutic choices before surgery. For instance, patients with a higher LMR might achieve sufficient benefits from curative intent policy and radical hepatectomy, and the following adjuvant chemotherapy administration was considered as the optimal therapeutic strategy. On the contrary, for patients with a lower LMR, hepatectomy may be ill-advised and further systemic therapy would be preferable. As mentioned earlier, this study further demonstrated that the LMR was more feasible for prognostic prediction in several specific subgroups. For example, in a male patient with colon cancer and primary lymph node metastases, the LMR might be more powerful to distinguish the prognosis. Because of the strengths of LMR, such as reliability, reproducibility, and low cost, we believed that it is easy to be applied in clinical practice and provided a supplementary diagnosis for the patients.
This study has several limitations. First, this is a retrospective study with uncontrolled methodology and a limited number of patients from a single-institution experience. Second, the preoperative treatment is inconsistent in some patients, which might affect inflammation-based index evaluation and survival outcomes. Additionally, complete data on the postoperative treatments of patients, such as regimen chemotherapies, types of radiotherapy, and interventional therapy, were not available. Also, subsequent therapies after disease recurrence play a critical role in prolonging the OS. Furthermore, appropriate cutoff levels of LMR, NLR, and PLR were calculated for OS using the ROC analysis. In fact, these cutoff variables were not consistent with those in previous studies and were necessary to be determined in validation studies. ${ }^{17,28}$ Finally, several disease conditions, such as infection, ischemia, diabetes mellitus, and heart disease, which may bias the blood-circulating cell counts, could not be taken into consideration. Overall, large prospective studies should be performed to confirm the findings of this study.

\section{Conclusion}

This study demonstrated that in patients with CLOM undergoing hepatic resection, preoperative LMR is the optimal and independent predictor of RFS and OS compared with the NLR and PLR. Thus, the LMR may help surgeons evaluate the benefit from curative hepatectomy and formulate individualized strategies of preoperative treatment.

\section{Acknowledgments}

We would like to thank all the colleagues of the Department of Colorectal Surgery in Sun Yat-sen University Cancer Center who were involved in treating the patients of this study.

This work was supported by grants from Guangzhou Science and Technology Plan Projects (Health Medical Collaborative Innovation Program of Guangzhou; grant No 201400000001-4) and Sun Yat-sen University Clinical Research 5010 Program (2015024); Science and Technology Planning Project of Guangdong Province (No 2013B0218001462).

\section{Disclosure}

The authors report no conflicts of interest in this work. The authenticity of this article has been validated by uploading the key raw data onto the Research Data Deposit public platform (www.researchdata.org.cn), with the approval number as RDDA2017000107. 


\section{References}

1. Chen W, Zheng R, Baade PD, et al. Cancer statistics in China, 2015. CA Cancer J Clin. 2016;66(2):115-132.

2. Van Cutsem E, Nordlinger B, Cervantes A; ESMO Guidelines Working Group. Advanced colorectal cancer: ESMO Clinical Practice Guidelines for treatment. Ann Oncol. 2010;21(Suppl 5):v93-v97.

3. O'Reilly DA, Poston GJ. Colorectal liver metastases: current and future perspectives. Future Oncol. 2006;2(4):525-531.

4. Akgul O, Cetinkaya E, Ersoz S, et al. Role of surgery in colorectal cancer liver metastases. World J Gastroenterol. 2014;20(20):6113-6122.

5. Primrose JN. Surgery for colorectal liver metastases. $\mathrm{Br} J$ Cancer. 2010;102(9):1313-1318

6. Vigano L, Capussotti L, Lapointe R, et al. Early recurrence after liver resection for colorectal metastases: Risk factors, prognosis, and treatment. A LiverMetSurvey-based study of 6,025 patients. Ann Surg Oncol. 2014;21(4):1276-1286.

7. Chan KM, Chiang JM, Lee CF, et al. Outcomes of resection for colorectal cancer hepatic metastases stratified by evolving eras of treatment. World J Surg Oncol. 2011;9:174.

8. Zlobec I, Lugli A. Prognostic and predictive factors in colorectal cancer. J Clin Pathol. 2008;61(5):561-569.

9. Mehrkhani F, Nasiri S, Donboli K, Meysamie A, Hedayat A. Prognostic factors in survival of colorectal cancer patients after surgery. Colorectal Dis. 2009;11(2):157-161.

10. Diakos CI, Charles KA, McMillan DC, Clarke SJ. Cancer-related inflammation and treatment effectiveness. Lancet Oncol. 2014;15(11): e493-e503.

11. Liu J, Geng Q, Chen S, et al. Nomogram based on systemic inflammatory response markers predicting the survival of patients with resectable gastric cancer after D2 gastrectomy. Oncotarget. 2016;7(25): 37556-37565.

12. Roxburgh CS, McMillan DC. Role of systemic inflammatory response in predicting survival in patients with primary operable cancer. Future Oncol. 2010;6(1):149-163.

13. Chua W, Charles KA, Baracos VE, Clarke SJ. Neutrophil/lymphocyte ratio predicts chemotherapy outcomes in patients with advanced colorectal cancer. Br J Cancer. 2011;104(8):1288-1295.

14. You J, Zhu GQ, Xie L, et al. Preoperative platelet to lymphocyte ratio is a valuable prognostic biomarker in patients with colorectal cancer. Oncotarget. 2016;7(18):25516-25527.

15. Stotz M, Pichler M, Absenger G, et al. The preoperative lymphocyte to monocyte ratio predicts clinical outcome in patients with stage III colon cancer. Br J Cancer. 2014;110(2):435-440.

16. Neofytou K, Smyth EC, Giakoustidis A, Khan AZ, Cunningham D, Mudan S. Elevated platelet to lymphocyte ratio predicts poor prognosis after hepatectomy for liver-only colorectal metastases, and it is superior to neutrophil to lymphocyte ratio as an adverse prognostic factor. Med Oncol. 2014;31(10):239.

17. Neofytou K, Smyth EC, Giakoustidis A, et al. The preoperative lymphocyte-to-monocyte ratio is prognostic of clinical outcomes for patients with liver-only colorectal metastases in the neoadjuvant setting. Ann Surg Oncol. 2015;22(13):4353-4362.

18. Fong Y, Fortner J, Sun RL, Brennan MF, Blumgart LH. Clinical score for predicting recurrence after hepatic resection for metastatic colorectal cancer: analysis of 1001 consecutive cases. Ann Surg. 1999; 230(3):309-318, discussion 318-321.

OncoTargets and Therapy

\section{Publish your work in this journal}

OncoTargets and Therapy is an international, peer-reviewed, open access journal focusing on the pathological basis of all cancers, potential targets for therapy and treatment protocols employed to improve the management of cancer patients. The journal also focuses on the impact of management programs and new therapeutic agents and protocols on

Submit your manuscript here: http://www.dovepress.com/oncotargets-and-therapy-journal
19. Lu Z, Peng J, Wang Z, et al. High preoperative serum CA19-9 level is predictive of poor prognosis for patients with colorectal liver oligometastases undergoing hepatic resection. Med Oncol. 2016;33(11):121.

20. Schwarz L, Michel P, Scotte M. Predictive factors for the benefit of perioperative FOLFOX for resectable liver metastasis in colorectal cancer patients (EORTC Intergroup Trial 40983). Ann Surg. 2015;261(1):e28-e29.

21. Colotta F, Allavena P, Sica A, Garlanda C, Mantovani A. Cancer-related inflammation, the seventh hallmark of cancer: links to genetic instability. Carcinogenesis. 2009;30(7):1073-1081.

22. Park JH, Watt DG, Roxburgh CS, Horgan PG, McMillan DC. Colorectal cancer, systemic inflammation, and outcome: Staging the tumor and staging the host. Ann Surg. 2016;263(2):326-336.

23. Shibutani M, Maeda K, Nagahara H, et al. Significance of markers of systemic inflammation for predicting survival and chemotherapeutic outcomes and monitoring tumor progression in patients with unresectable metastatic colorectal cancer. Anticancer Res. 2015;35(9):5037-5046.

24. Gu L, Li H, Chen L, et al. Prognostic role of lymphocyte to monocyte ratio for patients with cancer: Evidence from a systematic review and meta-analysis. Oncotarget. 2016;7(22):31926-31942.

25. Chan JC, Chan DL, Diakos CI, et al. The Lymphocyte-to-Monocyte ratio is a superior predictor of overall survival in comparison to established biomarkers of resectable colorectal cancer. Ann Surg. 2017;265(3): 539-546.

26. Shibutani M, Maeda K, Nagahara H, et al. Prognostic significance of the lymphocyte-to-monocyte ratio in patients with metastatic colorectal cancer. World J Gastroenterol. 2015;21(34):9966-9973.

27. Lin GN, Liu PP, Liu DY, Peng JW, Xiao JJ, Xia ZJ. Prognostic significance of the pre-chemotherapy lymphocyte-to-monocyte ratio in patients with previously untreated metastatic colorectal cancer receiving FOLFOX chemotherapy. Chin J Cancer. 2016;35:5.

28. Ozawa T, Ishihara S, Kawai K, et al. Impact of a lymphocyte to monocyte ratio in stage IV colorectal cancer. J Surg Res. 2015;199(2):386-392.

29. Lin EY, Pollard JW. Role of infiltrated leucocytes in tumor growth and spread. Br J Cancer. 2004;90(11):2053-2058.

30. Dunn GP, Old LJ, Schreiber RD. The immunobiology of cancer immunosurveillance and immunoediting. Immunity. 2004;21(2):137-148.

31. Menges T, Engel J, Welters I, et al. Changes in blood lymphocyte populations after multiple trauma: Association with posttraumatic complications. Crit Care Med. 1999;27(4):733-740.

32. Liang L, Zhu J, Jia H, et al. Predictive value of pretreatment lymphocyte count in stage II colorectal cancer and in high-risk patients treated with adjuvant chemotherapy. Oncotarget. 2016;7(1):1014-1028.

33. Qian BZ, Li J, Zhang H, et al. CCL2 recruits inflammatory monocytes to facilitate breast-tumor metastasis. Nature. 2011;475(7355):222-225.

34. Movahedi K, Laoui D, Gysemans C, et al. Different tumor microenvironments contain functionally distinct subsets of macrophages derived from Ly6C(high) monocytes. Cancer Res. 2010;70(14):5728-5739.

35. Condeelis J, Pollard JW. Macrophages: Obligate partners for tumor cell migration, invasion, and metastasis. Cell. 2006;124(2):263-266.

36. Rogers TL, Holen I. Tumor macrophages as potential targets of bisphosphonates. J Transl Med. 2011;9:177.

37. Di Caro G, Cortese N, Castino GF, et al. Dual prognostic significance of tumor-associated macrophages in human pancreatic adenocarcinoma treated or untreated with chemotherapy. Gut. 2016;65(10):1710-1720.

38. Steidl C, Lee T, Shah SP, et al. Tumor-associated macrophages and survival in classic Hodgkin's lymphoma. N Engl J Med. 2010;362(10): 875-885.

\section{Dovepress}

patient perspectives such as quality of life, adherence and satisfaction. The manuscript management system is completely online and includes a very quick and fair peer-review system, which is all easy to use. Visit http://www.dovepress.com/testimonials.php to read real quotes from published authors. 